\title{
Early postoperative outcomes of a novel nonexcisional technique using aluminum potassium sulfate and tannic acid sclerotherapy with mucopexy on patients with grade III hemorrhoids
}

\author{
Shunya Takada, Akira Tsunoda, Tomoko Takahashi, Hiroshi Kusanagi \\ Department of Gastroenterological Surgery, Kameda Medical Center, Kamogawa, Japan
}

Purpose: Aluminum potassium sulfate and tannic acid (ALTA; Zion, Mitsubishi Pharma Corp.) is an effective sclerosing agent for internal hemorrhoids. ALTA therapy with a rectal mucopexy (AM) is a new approach for treating hemorrhoidal prolapse. This study compared the early postoperative outcomes of AM surgery with Doppler-guided transanal hemorrhoidal dearterialization and mucopexy (DM) in patients with third-degree hemorrhoids.

Methods: AM surgery was performed on 32 patients with grade III hemorrhoids and was compared with a cohort of 22 patients who underwent DM surgery in a previous randomized controlled trial.

Results: The pain scores during defecation were significantly lower in the AM patients beginning 4 days after surgery. The total use of analgesics 2 weeks postoperatively was significantly lower in the AM patients than in the DM patients (3.5 tablets [range 1.6-5.5] vs. 7.6 tablets [range 3.3-11.9], $\mathrm{P}=0.04$ ). The length of operation, blood loss, and incidence of postoperative complications were significantly lower in the AM patients than in the DM patients. During 12 months follow-up, recurrence of prolapse occurred in 1 patient who underwent AM surgery.

Conclusion: AM surgery is effective, with lower complication rates and postoperative analgesic requirements, and is a less invasive treatment for patients with grade III hemorrhoids compared to DM surgery.

Keywords: Hemorrhoids; Prolapse; Aluminum potassium sulfate and tannic acid; Transanal hemorrhoidal dearterialization; Mucopexy

\section{INTRODUCTION}

Hemorrhoidectomy is considered the gold standard for the treatment of advanced hemorrhoids because of the low recurrence rates, even if it is not without complications and can be associated with considerable pain [1]. In an attempt to relieve the postopera-

Received: Oct 27, 2020 - Revised: Feb 9, 2021 - Accepted: Apr 13, 2021 Correspondence to: Shunya Takada, M.D.

Department of Gastroenterological Surgery, Kameda Medical Center, 929 Higashi-cho, Kamogawa, Chiba 296-8602, Japan

Tel: +81-4-7092-2211, Fax: +81-4-7099-1198

E-mail: takada.shunya@kameda.jp

ORCID: https://orcid.org/0000-0002-8691-7658

(C) 2022 The Korean Society of Coloproctology

This is an open-access article distributed under the terms of the Creative Commons Attribution NonCommercial License (https://creativecommons.org/licenses/by-nc/4.0) which permits unrestricted noncommercial use, distribution, and reproduction in any medium, provided the original work is properly cited. tive pain, Morinaga et al. [2] developed a new nonexcisional procedure for hemorrhoids described as Doppler-guided hemorrhoidal artery ligation or transanal hemorrhoidal dearterialization (THD). Previous studies reported that this technique was associated with less postoperative pain than standard hemorrhoidectomy [3-5]. We agreed with the view that additional mucopexy may reduce prolapse of hemorrhoids [6]; thus, we have performed the THD procedure with mucopexy (DM) since $2012[7,8]$. However, the Doppler-guided proctoscope was expensive, and the DM surgery was performed at the cost of $€ 300$ per patient [7, 8]. This underscores the need for a novel technique that is less expensive than the DM surgery and can achieve postoperative outcomes comparable to or better than DM surgery.

A novel sclerosing agent containing aluminum potassium sulfate and tannic acid (ALTA) has been developed in Japan. The ALTA injection therapy for hemorrhoids proved to be effective 
for both bleeding and prolapse $[9,10]$. Success rates of $75 \%$ to $93 \%$ for grade III hemorrhoids at 1-year have been reported [11, 12]. We hypothesized that additional mucopexy with the ALTA therapy may reduce prolapse of hemorrhoids, in the same way as the THD procedure with mucopexy decreased prolapse compared with the THD procedure alone [6]. Applying the hypothesis, we have performed the ALTA injection with mucopexy (AM) since 2018. The aim of this study was to compare the short-term outcomes of AM surgery on patients with grade III hemorrhoids with that of DM surgery from our previous study [7]. We evaluated the impact of AM and DM surgery on postoperative pain scores and analgesic requirements.

\section{METHODS}

A total of 32 patients with symptomatic grade III hemorrhoids were recruited to undergo AM surgery from April 2018 to March 2020 in Kameda Medical Center, Kamogawa, Japan. The Institutional Review Board of Kameda Medical Center approved this study (No. 20-022), and all patients gave written informed consent. Patients who did not provide written informed consent were excluded. The exclusion criteria included thrombosed hemorrhoids, anal fissures, anal fistulas, anal polyps, anorectal mucosal prolapse, pregnancy, continuous analgesic use, inflammatory bowel diseases, paradoxical puborectalis contraction, anal stenosis, and bleeding disorder. Patients who had undergone rubber band ligation or sclerotherapy in the past 6 months, hemorrhoidectomy within 5 years, or any previous operation with hemorrhoidal dearterialization or stapled anopexy were also excluded.

These patients were compared with a cohort of 22 patients treated with DM surgery in a previous study [7], so the study design was mixed with previously reported cohort data and retrospectively collected recent data.

The primary endpoints included postoperative pain scores and analgesic requirements. The secondary endpoints included postoperative complications, resumption of work or normal daily activities, length of operation, blood loss, hospital stay, recurrence rate at 1 year, and patient satisfaction score. Patient demographics and symptoms were recorded. Anal incontinence was evaluated using the Fecal Incontinence Severity Index (FISI) [13].

\section{Surgical technique \\ AM surgery}

Patients were placed in the jack-knife position under spinal anesthesia. A proctoscope designed to offer an open superior window to expose the entire operative field was used for the prolapsing hemorrhoids requiring mucopexy. Using a continuous suture, mucopexy was performed starting at 6 to $7 \mathrm{~cm}$ from the anal verge until the anorectal junction, $1 \mathrm{~cm}$ above the dentate line. Within $2 \mathrm{~cm}$ from the anorectal junction, transfixing the rectum was undertaken to include the possible existence of submucosal hemorrhoidal arteries [2]. Care was observed to avoid suturing of the internal and external piles. The suture was tied to complete mucopexy. ALTA solution was then injected into prolapsing hemorrhoid according to the 4-step injection method (Fig. 1) [1]. ALTA solution was also injected into the nonprolapsing hemorrhoids. The number of hemorrhoids undergoing mucopexy and/ or ALTA therapy, and the total volume of ALTA solution injected were recorded.

\section{DM surgery}

Doppler-guided hemorrhoidal dearterialization was carried out using THD easy model (S.P.A., Correggio, Italy), which has been described in detail elsewhere [7]. The Doppler proctoscope was inserted through the anal canal to the lower rectum. Tilting the proctoscope, the best Doppler signals were sought that correspond to all 6 main trunks of the hemorrhoidal arteries. Moving the proctoscope distally, every arterial branch signal was followed in a craniocaudal direction, until the best Doppler signal was obtained at about $2 \mathrm{~cm}$ above the anorectal junction. For the sites undergoing dearterialization alone, a hemostatic Z-shaped suture was placed and tied. For the prolapsing hemorrhoids requiring mucopexy, after arterial identification, a small mark was made using electrocautery on the mucosa at the maximal signal. A similar figured proctoscope used for mucopexy in AM surgery was used. Using a continuous running suture, mucopexy was done starting at 6 to $7 \mathrm{~cm}$ from the anal verge until the previously marked point. A suture was then inserted above and below this point. The mucopexy was completed at the anorectal junction, and the suture was tied.

\section{Follow-up}

The postoperative care and follow-up were in accordance with

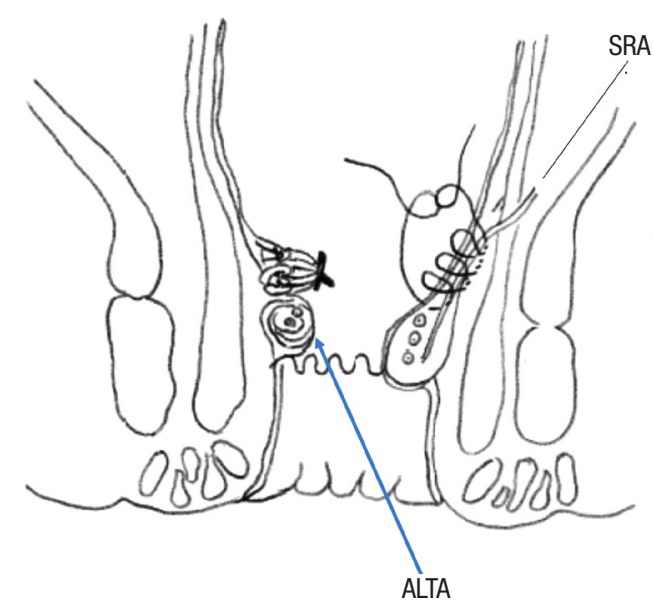

Fig. 1. Schema of the aluminum potassium sulfate and tannic acid (ALTA) injection with mucopexy surgery. Using a continuous suture, mucopexy is performed starting at 6 to $7 \mathrm{~cm}$ from the anal verge until the anorectal junction. The suture is tied, then ALTA is injected into hemorrhoid. SRA, suprarectal artery. 
those done in the previous study [7]. A stool softener was prescribed, and patients were advised to avoid straining, while a normal diet was allowed. For analgesics, Loxonine (Daiichi Sankyo Corp., Tokyo, Japan) (1 tablet, 4 times a day as needed) was given orally. The criteria for the patient's discharge from the hospital were (1) no fever, and (2) no significant complications. The decision of the timing for discharge was made on the morning the day after surgery.

Pain scores at rest and the pain scores during defecation on postoperative days 1 to 7 and day 14 , and the analgesic requirements on postoperative days 1 to 14 were reported. Pain assessment was based on a numeral rating scale $(0$, no pain at all; 10 , worst pain imaginable). The length of operation, blood loss, and length of hospital stay were also recorded. All postoperative complications were assessed using the Clavien-Dindo (CD) classification system [14]. Patients were followed up at the clinic 1, 2, and 3 months after surgery, and late complications were noted. After AM surgery, patients were instructed to report their symptoms annually by mail. The final follow-up for patients who underwent DM surgery was conducted in June 2020, and similarly, the patients were instructed to report their symptoms by mail. Those who indicated that they had a feeling of prolapse were examined in the clinic. At 12 months, patients' satisfaction scores were also recorded by using a numeral rating scale $(0$, no satisfaction at all; 10 , most satisfied). The FISI score was recorded at baseline, 1, and 3 months. All patients were submitted to anoscopy at 2 to 3 months after surgery.

\section{Statistical analysis}

All statistical analyses were performed with EZR (ver. 1.54, free statistical software; Kanda Y, Saitama Medical Center, Jichi Medical University, Saitama, Japan [15]) which is a graphical user interface for EZR. More precisely, it is a modified version of R commander designed to add statistical functions frequently used in

Table 1. Demographic data

\begin{tabular}{lccc}
\hline Variable & AM & DM & P-value \\
\hline No. of patients & 32 & 22 & \\
Mean age (yr) & $70(27-6)$ & $57(25-81)$ & $<0.0001^{\mathrm{a}}$ \\
Sex (male:female) & $24: 8$ & $19: 3$ & $0.49^{\mathrm{b}}$ \\
Previous procedure $^{\mathrm{c}}$ & $13(40.6)$ & $1(4.5)$ & $0.004^{\mathrm{b}}$ \\
Hemorrhoidectomy $^{\mathrm{b}}$ & 7 & 1 & \\
ALTA therapy & 3 & & \\
Laser therapy & 2 & & \\
Rubber band ligation & 1 & & \\
\hline
\end{tabular}

Values are presented as number only, mean (range), or number (\%). AM, ALTA injection with mucopexy; DM, transanal hemorrhoidal dearterialization with mucopexy; ALTA, aluminum potassium sulfate and tannic acid.

aMann-Whitney U-test. ${ }^{b}$ chi-square test. ${ }^{\text {PPerformed mean }}$ (95\% confidence interval) duration of 16.2 years (7.9-24.5 years) before the surgeries in this study. biostatistics. Continuous variables were analyzed by using the Mann-Whitney U-test, and categorical variables were analyzed by the chi-square test. The results are expressed as the means (95\% confidence interval, CI) unless otherwise specified. A P-value of $<0.05$ was considered to be statistically significant.

\section{RESULTS}

We included 32 patients who underwent AM surgery; compared to the 22 patients who underwent DM surgery. The patients in the AM group were significantly older than those in the DM group. More patients in the AM group had undergone previous surgeries for hemorrhoids than those in the DM group. These patients were included in the study because they had undergone the previous surgeries at least 5 years (mean, 16.2 years; 95\% CI, 7.924.5 years) before the surgeries in this study (Table 1 ).

The length of operation and blood loss were significantly lower in the AM patients than that in the DM patients. The number of mucopexy procedures, hospital stay, and time until either the first defecation after surgery or the resumption of normal daily activity was not significantly different between the 2 groups (Table 2).

The postoperative pain scores at rest revealed that significantly less discomfort was noted on days 5 and 7 in the AM patients than in the DM patients (Fig. 2). The pain scores during defecation were significantly lower in the AM patients for 5 days during the first week (Fig. 3). The total dose of analgesics during the first 2 weeks after surgery was significantly lower in the AM patients than in the DM patients (Fig. 4). Fewer analgesics were required after surgery in the AM patients compared to the DM patients (17 of 22 [77.3\%] vs. 17 of 32 [53.1\%], $\mathrm{P}=0.09$ ).

The postoperative complications are listed in Table 3. Intraoper-

Table 2. Early postoperative results

\begin{tabular}{|c|c|c|c|}
\hline Variable & $\mathrm{AM}(\mathrm{n}=32)$ & $\mathrm{DM}(\mathrm{n}=22)$ & $P$-value ${ }^{a}$ \\
\hline Hemorrhoidal dearterialization $^{\mathrm{b}}$ & & $5.8(4-6)$ & \\
\hline ALTA procedures ${ }^{b}$ & $2.7(1-3)$ & & \\
\hline Mucopexy procedures ${ }^{b}$ & $1.8(1-3)$ & $2.0(1-4)$ & 0.25 \\
\hline $\begin{array}{l}\text { Total dose of ALTA injection }{ }^{c} \\
(\mathrm{~mL})\end{array}$ & $18.2(17.3-19.0)$ & & \\
\hline Length of operation (min) & $24.5(22.0-26.9)$ & $35.9(32.7-39.0)$ & $<0.05$ \\
\hline Blood loss ${ }^{c}(\mathrm{~mL})$ & $4.7(3.1-6.4)$ & $13.2(3.1-23.3)$ & 0.007 \\
\hline Hospital stay (day) & $2.0(2.0-2.1)$ & $2.0(2.0-2.0)$ & 0.42 \\
\hline First defecation ${ }^{c}$ (POD) & $0.9(0.7-1.1)$ & $1.3(1.0-1.6)$ & 0.054 \\
\hline $\begin{array}{l}\text { Resumption of work or } \\
\text { normal daily activityc (POD) }\end{array}$ & $4.1(2.7-5.4)$ & $3.7(2.4-5.0)$ & 0.74 \\
\hline
\end{tabular}

Values are presented as ${ }^{\mathrm{b}}$ mean (range) or ${ }^{\mathrm{c}}$ mean (95\% confidence interval). AM, ALTA injection with mucopexy; DM, transanal hemorrhoidal dearterialization with mucopexy; ALTA, aluminum potassium sulfate and tannic acid; POD, postoperative day.

aMann-Whitney U-test. 


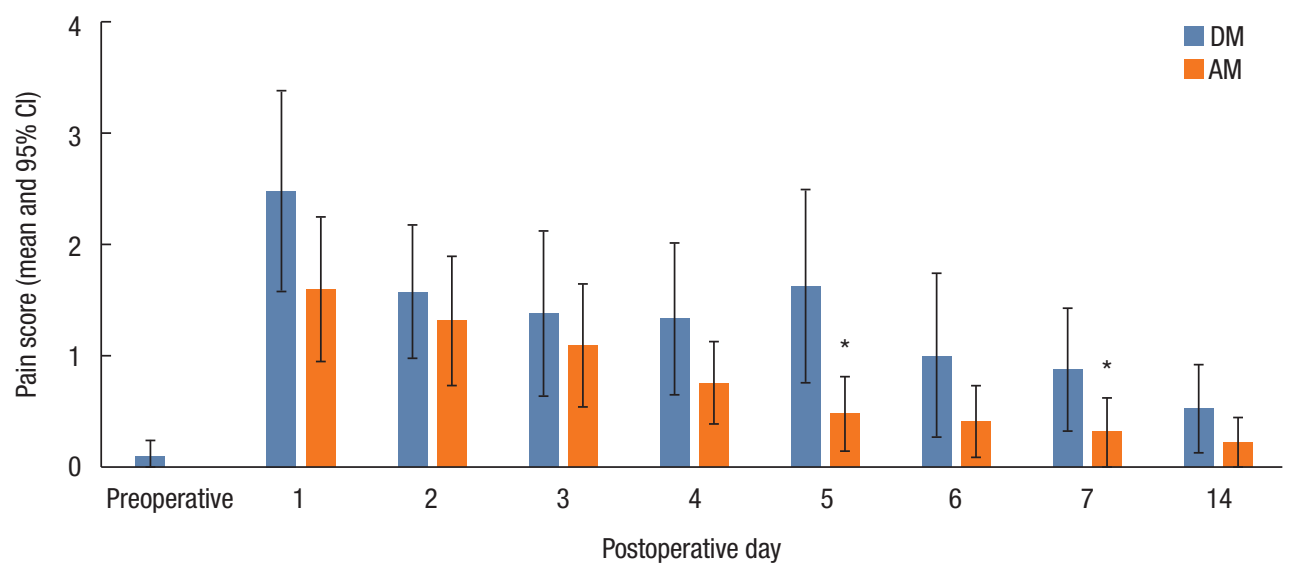

Fig. 2. Pain scores at rest. DM, transanal hemorrhoidal dearterialization with mucopexy; AM, aluminum potassium sulfate and tannic acid injection with mucopexy; CI, confidence interval. ${ }^{\star} \mathrm{P}<0.05$ (comparing between AM and DM; Mann-Whitney U-test).

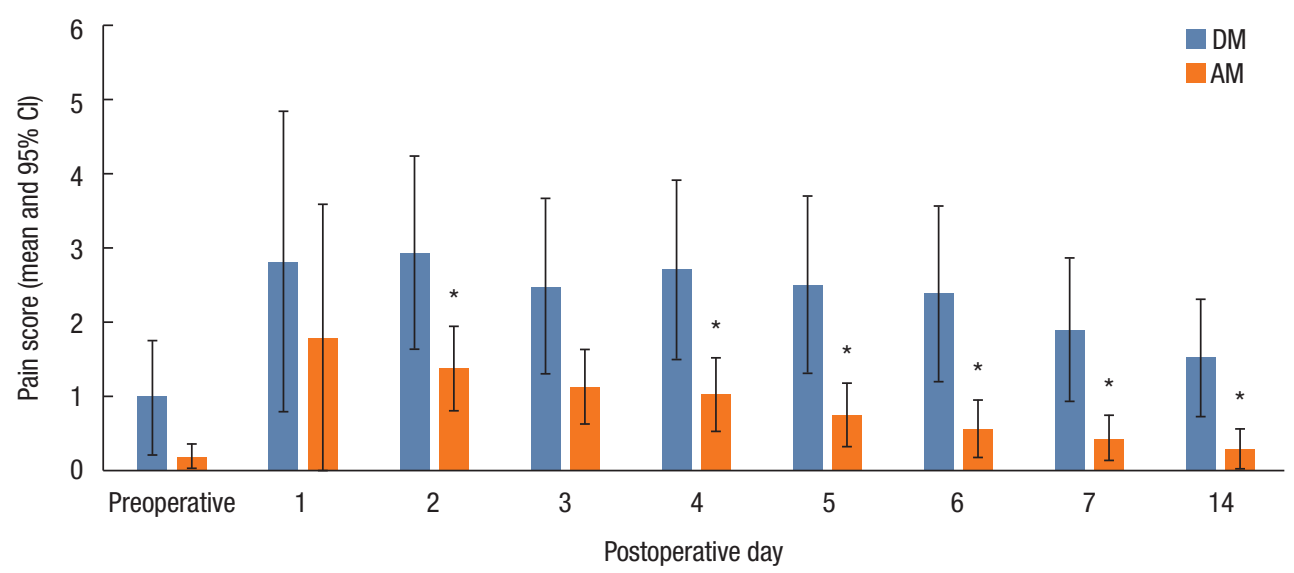

Fig. 3. Pain scores during defecation. DM, transanal hemorrhoidal dearterialization with mucopexy; AM, aluminum potassium sulfate and tannic acid injection with mucopexy; CI, confidence interval. ${ }^{\star} \mathrm{P}<0.05$ (comparing between AM and DM; Mann-Whitney U-test).

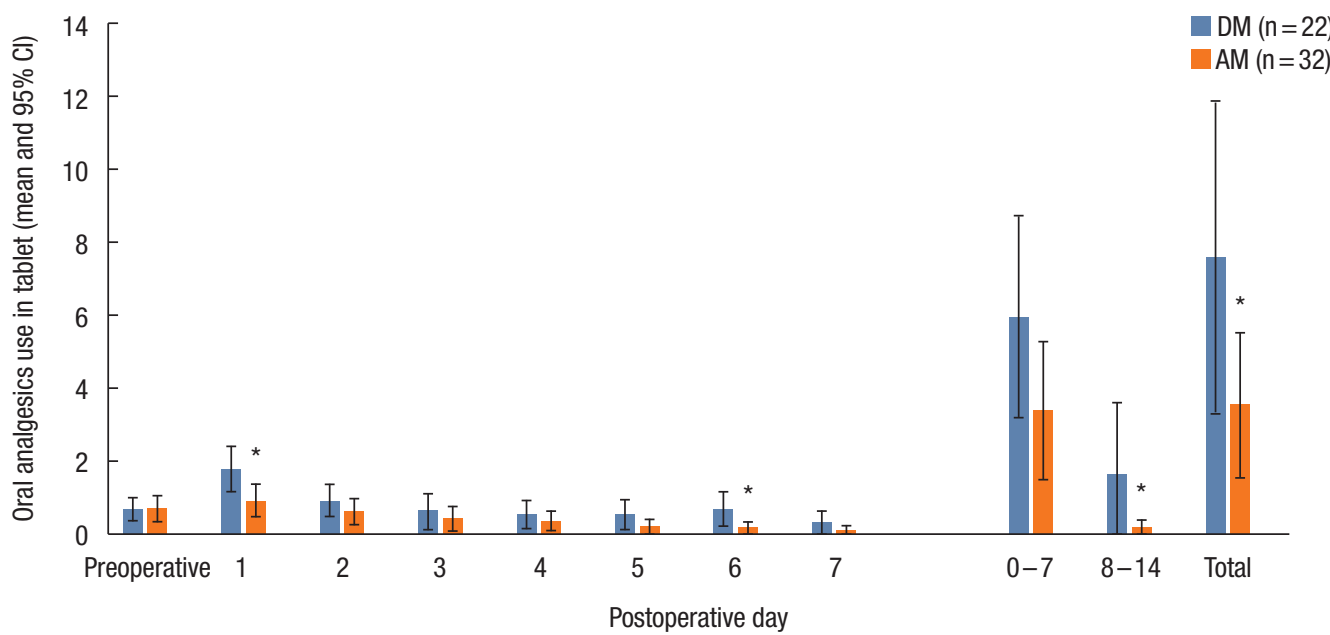

Fig. 4. Oral analgesics use (tablets). DM, transanal hemorrhoidal dearterialization with mucopexy; AM, aluminum potassium sulfate and tannic acid injection with mucopexy; CI, confidence interval. ${ }^{\star} \mathrm{P}<0.05$ (comparing between $\mathrm{AM}$ and DM; Mann-Whitney U-test). 
ative transitory submucosal hematomas developed in 1 patient who had DM surgery. Urinary retention occurred in 3 patients in the DM group (13.6\%) and 1 patient in the AM group (3.1\%). Postoperative hemorrhage necessitating readmission was noted in 1 patient (4.5\%) in the DM group, which resolved spontaneously. Thrombosed residual hemorrhoids developed in 3 patients in the DM group (13.6\%) and 1 patient in the AM group (3.1\%). None of the patients in either group complained of anal stenosis at the 3-month follow-up. The incidence of CD grade I complications and total complications was significantly lower after AM surgery than DM surgery. CD grade II or IIIa complications did not occur in the AM patients.

Each hemorrhoidal symptom was examined separately. Hemorrhage, pain, and the need for manual repositioning of hemorrhoids were all improved after surgery in both groups of patients at 1 and 3 months (Table 4). Anoscopy at 2 to 3 months did not

Table 3. The complications according to Clavien-Dindo classification

\begin{tabular}{lccc}
\hline Variable & AM $(\mathrm{n}=32)$ & $\mathrm{DM}(\mathrm{n}=22)$ & P-value $^{\mathrm{d}}$ \\
\hline Total complications & $1(3.1)$ & $7(31.8)$ & 0.006 \\
Clavien-Dindo grade $^{\mathrm{a}}$ & & & \\
I & $1(3.1)$ & $6(27.3)$ & 0.014 \\
$\|$ & $0(0)$ & $0(0)$ & $\mathrm{NA}$ \\
$\quad \|^{\mathrm{a}}{ }^{\mathrm{a}}$ & 0 & $1(4.5)$ & 0.41 \\
Complication type & & & \\
Urinary retention & $1(3.1)^{\mathrm{b}}$ & $3(13.6)$ & 0.29 \\
Secondary hemorrhage & $0(0)$ & $1(4.5)^{\mathrm{c}}$ & 0.41 \\
Thrombosed residual hemorrhoid & $1(3.1)^{\mathrm{b}}$ & $3(13.6)^{\mathrm{d}}$ & 0.29 \\
Anal stenosis at 3 months & $0(0)$ & $0(0)$ & $\mathrm{NA}$ \\
\hline
\end{tabular}

Values are presented as number (\%).

AM, aluminum potassium sulfate and tannic acid injection with mucopexy; DM, transanal hemorrhoidal dearterialization with mucopexy; NA, not applicable.

${ }^{\text {aF }}$ or overall complications. ${ }^{b}$ Same patient, ${ }^{\circ}$ needed readmission, done underwent reoperation. ${ }^{\mathrm{d}}$ Chi-square test. show prolapsed hemorrhoids in either group. There were no significant differences between the groups in the mean postoperative FISI scores at 1 and 3 months (Table 5), and no significant difference in mean patient satisfaction scores at 12 months (8.8 [8.59.2] in AM group vs. 7.1 [5.6-8.7] in DM group, $\mathrm{P} \geq 0.05$ ).

The median (range) of follow-up was 44 months (20-79 months) in the DM group and 12 months (12-26 months) in the AM group. The DM group reported 5 patients with a prolapsed hemorrhoid. Three patients had additional surgery; 2 had hemorrhoidectomy, and 1 had AM surgery. In contrast, 1 patient reported a prolapsed hemorrhoid 8 months after surgery in the AM group, which was diagnosed as grade III hemorrhoids at the clinic. He was scheduled to undergo AM surgery.

\section{DISCUSSION}

Our study compared the postoperative results of patients with hemorrhoids who underwent AM and DM surgery. This study demonstrated that 2 weeks after surgery, the AM patients needed significantly lower analgesic tablets than the DM patients. The AM patients complained of significantly less pain during defecation beginning 4 days after surgery than the DM patients. The length of operation, blood loss, and the incidence of postoperative complications were significantly lower in the AM patients than that in the DM patients, respectively. Recurrence of prolapse occurred in 1 patient who underwent AM surgery during 12

Table 5. Fecal Incontinence Severity Index score in 2 groups

\begin{tabular}{lccc}
\hline Time & AM $(\mathrm{n}=32)$ & $\mathrm{DM}(\mathrm{n}=22)$ & P-value $^{\mathrm{a}}$ \\
\hline Preoperative & $0.7(-0.1$ to 1.6$)$ & $1.1(-0.3$ to 2.5$)$ & 0.61 \\
Postoperative (mo) & & & \\
1 & $0.8(-0.2$ to 1.9$)$ & $0.3(-0.3$ to 0.8$)$ & 0.50 \\
3 & $0.6(-0.1$ to 1.4$)$ & $1.1(-0.6$ to 2.8$)$ & 0.97
\end{tabular}

$\mathrm{AM}$, aluminum potassium sulfate and tannic acid injection with mucopexy; DM, transanal hemorrhoidal dearterialization with mucopexy.

aMann-Whitney U-test.

Table 4. Number of patients reporting each symptom once a week or more preoperatively, at follow-up after 1 and 3 months

\begin{tabular}{|c|c|c|c|c|c|c|}
\hline \multirow{3}{*}{ Symptom } & \multirow{2}{*}{\multicolumn{2}{|c|}{ Preoperative }} & \multicolumn{4}{|c|}{ Postoperative (mo) } \\
\hline & & & \multicolumn{2}{|c|}{1} & \multicolumn{2}{|c|}{3} \\
\hline & $\mathrm{AM}(\mathrm{n}=32)$ & $\mathrm{DM}(\mathrm{n}=22)$ & $\operatorname{AM}(n=32)$ & $\mathrm{DM}(\mathrm{n}=22)$ & $\mathrm{AM}(\mathrm{n}=32)$ & $\mathrm{DM}(\mathrm{n}=22)$ \\
\hline Pain & 13 & 7 & $0^{\mathrm{a}}$ & $1^{\mathrm{a}}$ & $0^{\mathrm{a}}$ & $0^{\mathrm{a}}$ \\
\hline Mucus discharge & 5 & 4 & 1 & 1 & 0 & 0 \\
\hline Manual reposition & 32 & 22 & $0^{\mathrm{a}}$ & $0^{\mathrm{a}}$ & $0^{\mathrm{a}}$ & $0^{\mathrm{a}}$ \\
\hline
\end{tabular}

AM, aluminum potassium sulfate and tannic acid injection with mucopexy; DM, transanal hemorrhoidal dearterialization with mucopexy.

ap $<0.01$ (comparing between preoperative and postoperative symptom; chi-square test). 
months follow-up.

Early prolapse was not found after surgery in each group. In DM patients, the vascular inflow to the hemorrhoids decreases after successful ligation of the arteries, while the venous outflow remains intact; thus shrinking the hemorrhoids. On the contrary, in AM patients, the active ingredient, alum, reduces blood flow in hemorrhoids, reducing hemorrhoid size, which then induces chronic inflammation causing fibrosis. The adjunct ingredient, tannic acid, reduces excessive acute inflammation induced by alum and has a strong astringent effect on tissues, promoting protein coagulation and concentration of blood vessels, while decreasing exudation into tissues from the inflammatory reaction. These cause sclerosis and retraction of the hemorrhoids $[16,17]$. Adverse effects of the ALTA therapy included pyrexia, anal pain, and rectal ulcer $[9,12]$, where pyrexia was the most frequent reaction with an incidence of 9\% [9], but it did not occur in the AM patients. Mucopexy performed in either group may improve the venous drainage from the hemorrhoidal plexus because of mucosal sliding that may impair venous drainage [18].

There were no significant intraoperative complications that occurred in either group. One patient in each group developed transient submucosal hematomas, none of which caused any clinical adverse effects. While no serious early and delayed complications were noted in either group, the $\mathrm{CD}$ grade II or IIIa complications did not occur after AM surgery, and the incidence of total complications was significantly lower in the AM patients than that in the DM patients. These findings suggest that AM surgery may be less invasive than DM surgery. The blood loss was greater in the DM patients because the hemorrhoidal arteries (nearly 6 in total) were treated by placing transfixing sutures into the submucosal layer around the lower rectum, which led to increased hemorrhage in the DM patients. No postoperative bleeding requiring readmission occurred in the AM patients. Functionally, there was no change from the preoperative FISI scores in either group. Restoring the hemorrhoidal cushions to their original sites, rather than excising them, has the advantage of restoring the physiological role of these structures in the mechanism of anal continence.

Recurrence of prolapse occurred in 5 patients $(22.7 \%)$ who underwent the DM surgery during a 44-month follow-up. In a systematic review including 28 studies, the pooled recurrence rate of Doppler-guided dearterialization was $17.5 \%$, with a wide range of $3 \%$ to $60 \%$, due to the variability of preoperative hemorrhoidal grade or additional procedures such as mucopexy being performed simultaneously [19]. A multicenter trial reported recurrence rates of $9.7 \%$ for grade III $(n=548)$ and $10.2 \%$ for grade IV $(\mathrm{n}=118)$ at 11 months after the DM surgery [20]. Scheyer et al. [21] reported that $24.0 \%$ (98 of 408) of patients reported recurrent prolapsing piles at 6.5 years after the DM surgery. Recurrence of prolapse occurred in 1 patient (3\%) who underwent AM surgery during a 12-month follow-up in this study. The recurrence rates of sclerotherapy by ALTA alone for grade III hemorrhoids have been reported to be $7 \%$ to $25 \%$ at 1-year follow-up [11, 12].
Long-term follow-up is required to assess the recurrence after AM surgery.

More patients in the DM group required analgesics after surgery than those in the AM group. In either surgery, rectal mucopexy near the dentate line may have caused postoperative pain. In addition, dearterialization of 6 hemorrhoidal arteries in the DM patients may cause ischemia, possibly causing postoperative pain or rectal discomfort. Dearterialization of hemorrhoidal arteries may have occurred during mucopexy of the AM patients, but was fewer (approximately 2) than that in the DM patients. The added value of Doppler guidance for hemorrhoidal artery ligation has been questioned. Schuurman et al. [22] described a randomized study of 82 patients with grade II or III hemorrhoids comparing Doppler-guided ligation or ligation without Doppler. More patients in the Doppler group reported recurrence of prolapse at 6 months than those in the non-Doppler group. Postoperative pain was transient, and a majority of the patients in both groups did not require analgesics beginning 5 days after surgery ( 28 of 32 in AM group vs. 15 of 22 in DM group). A postoperative daily pain score after the DM surgery was scarcely assessed in the literature. The average pain scores of less than 4 were reported during the first 7 postoperative days by the patients who underwent the DM surgery in the study by Elmer et al. [23], which were similar results in this study. The pain scores were significantly lower in the AM patients for 2 days at rest and 5 days while defecating during the first week than in the DM patients, but the differences in the mean score were small (0.6-1.8 points) between the groups. As mentioned previously, the number of hemorrhoidal arteries ligated was lower in the AM patients than that in the DM patients, which may lead to less ischemia, causing postoperative lower pain scores in the AM patients.

The cost of DM surgery is approximately $€ 300$, while AM surgery costs $€ 40$ per patient. Clearly, AM surgery may be more costeffective than DM surgery.

This study has several limitations. First, this was not a randomized controlled trial. Second, the number of patients was small, and the follow-up period in the AM patients was short. Third, the clinical examination at follow-up was done by only one surgeon, who also performed the surgical procedures. Long-term results and recurrence rate of AM surgery should be evaluated in larger randomized controlled trials.

In conclusion, $\mathrm{AM}$ surgery may be the preferable nonexcisional surgery for third-degree hemorrhoids because it is safe and effective, with lower complication rates, less postoperative analgesic requirements, and cheaper compared to DM surgery.

\section{CONFLICT OF INTEREST}

No potential conflict of interest relevant to this article was reported. 


\section{FUNDING}

None.

\section{REFERENCES}

1. Jayaraman S, Colquhoun PH, Malthaner RA. Stapled versus conventional surgery for hemorrhoids. Cochrane Database Syst Rev 2006;18:CD005393.

2. Morinaga K, Hasuda K, Ikeda T. A novel therapy for internal hemorrhoids: ligation of the hemorrhoidal artery with a newly devised instrument (Moricorn) in conjunction with a Doppler flowmeter. Am J Gastroenterol 1995;90:610-3.

3. Sohn N, Aronoff JS, Cohen FS, Weinstein MA. Transanal hemorrhoidal dearterialization is an alternative to operative hemorrhoidectomy. Am J Surg 2001;182:515-9.

4. Felice G, Privitera A, Ellul E, Klaumann M. Doppler-guided hemorrhoidal artery ligation: an alternative to hemorrhoidectomy. Dis Colon Rectum 2005;48:2090-3.

5. Greenberg R, Karin E, Avital S, Skornick Y, Werbin N. First 100 cases with Doppler-guided hemorrhoidal artery ligation. Dis Colon Rectum 2006;49:485-9.

6. Dal Monte PP, Tagariello C, Sarago M, Giordano P, Shafi A, Cudazzo E, et al. Transanal haemorrhoidal dearterialisation: nonexcisional surgery for the treatment of haemorrhoidal disease. Tech Coloproctol 2007;11:333-8.

7. Tsunoda A, Takahashi T, Kusanagi H. A prospective randomized trial of transanal hemorrhoidal dearterialization with mucopexy versus ultrasonic scalpel hemorrhoidectomy for grade III hemorrhoids. Tech Coloproctol 2017;21:657-65.

8. Tsunoda A, Kiyasu Y, Fujii W, Kano N. Comparison of the early results of transanal hemorrhoidal dearterialization and hemorrhoidectomy using an ultrasonic scalpel. Surg Today 2015;45:17580.

9. Takano M, Iwadare J, Ohba H, Takamura H, Masuda Y, Matsuo K, et al. Sclerosing therapy of internal hemorrhoids with a novel sclerosing agent. Comparison with ligation and excision. Int J Colorectal Dis 2006;21:44-51.

10. Takamura H, Inatsugu H, Yoshikawa S, Masuda T. Treatment of internal hemorrhoids with Xiaozhiling injection. J Jpn Soc Coloproctol 2001;54:910-4.

11. Yano T, Yano K. Comparison of injection sclerotherapy between $5 \%$ phenol in almond oil and aluminum potassium sulfate and tannic acid for grade 3 hemorrhoids. Ann Coloproctol 2015;31:
103-5.

12. Miyamoto H, Hada T, Ishiyama G, Ono Y, Watanabe H. Aluminum potassium sulfate and tannic acid sclerotherapy for Goligher grades II and III hemorrhoids: results from a multicenter study. World J Hepatol 2016;8:844-9.

13. Rockwood TH, Church JM, Fleshman JW, Kane RL, Mavrantonis C, Thorson AG, et al. Patient and surgeon ranking of the severity of symptoms associated with fecal incontinence: the fecal incontinence severity index. Dis Colon Rectum 1999;42:1525-32.

14. Dindo D, Demartines N, Clavien PA. Classification of surgical complications: a new proposal with evaluation in a cohort of 6336 patients and results of a survey. Ann Surg 2004;240:205-13.

15. Kanda $Y$. Investigation of the freely available easy-to-use software 'EZR' for medical statistics. Bone Marrow Transplant 2013;48: 452-8.

16. Ono T, Goto K, Takagi S, Iwasaki S, Komatsu H. Sclerosing effect of OC-108, a novel agent for hemorrhoids, is associated with granulomatous inflammation induced by aluminum. J Pharmacol Sci 2005;99:353-63.

17. Ono T, Nakagawa H, Fukunari A, Hashimoto T, Komatsu H. Hemostatic action of OC-108, a novel agent for hemorrhoids, is associated with regional blood flow arrest induced by acute inflammation. J Pharmacol Sci 2006;102:314-20.

18. Thomson WH. The nature and cause of haemorrhoids. Proc R Soc Med 1975;68:574-5.

19. Pucher PH, Sodergren MH, Lord AC, Darzi A, Ziprin P. Clinical outcome following Doppler-guided haemorrhoidal artery ligation: a systematic review. Colorectal Dis 2013;15:e284-94.

20. Ratto C, Parello A, Veronese E, Cudazzo E, D’Agostino E, Pagano $\mathrm{C}$, et al. Doppler-guided transanal haemorrhoidal dearterialization for haemorrhoids: results from a multicentre trial. Colorectal Dis 2015;17:010-9.

21. Scheyer M, Antonietti E, Rollinger G, Lancee S, Pokorny H. Hemorrhoidal artery ligation (HAL) and rectoanal repair (RAR): retrospective analysis of 408 patients in a single center. Tech Coloproctol 2015;19:5-9.

22. Schuurman JP, Borel Rinkes IH, Go PM. Hemorrhoidal artery ligation procedure with or without Doppler transducer in grade II and III hemorrhoidal disease: a blinded randomized clinical trial. Ann Surg 2012;255:840-5.

23. Elmér SE, Nygren JO, Lenander CE. A randomized trial of transanal hemorrhoidal dearterialization with anopexy compared with open hemorrhoidectomy in the treatment of hemorrhoids. Dis Colon Rectum 2013;56:484-90. 